\title{
Article
}

\section{Sakiadis Flow of Harris Fluids: a Series-Solution}

\author{
Navid P. KHABAZI ${ }^{*}$, Mohsen ARYAN", Jalil JAMALI ${ }^{* *}$, and Kayvan SADEGHY ${ }^{* \dagger}$ \\ Center of Excellence in the Design and Optimization of Energy Systems (CEDOES) \\ "School of Mechanical Engineering, College of Engineering, University of Tehran, Iran \\ P.O.Box: 11155-4563,Tehran,Iran,sadeghy@ut.ac.ir \\ ${ }^{* *}$ Islamic Azad University, Shoushtar Branch, Shoushtar, Iran \\ (Received : May 2, 2014)
}

\begin{abstract}
In the present work, Sakiadis flow of a shear-thinning fluid obeying Harris rheological model is investigated analytically. Assuming that the flow is occurring at high Reynolds number, use will be made of the boundary layer theory to simplify the equations of motion. The equations so obtained are then reduced to a single fourth-order ODE using a suitable similarity variable. The homotopy analysis method (HAM) is used to solve the fourth-order nonlinear equation so obtained using the Mathematica software. The material parameters appearing in the Harris model are shown to be responsible for the lack of a self-similar solution, but fortunately the flow is shown to render itself to a local similarity solution. The results show that for the Harris model to represent shear-thinning fluids, the sign and magnitude of the material parameters appearing in this tricky rheological model should be carefully selected.
\end{abstract}

Key Words: Boundary layer / Homotopy analysis method (HAM) / Harris model / Sakiadis flow

\section{INTRODUCTION}

Boundary layer theory is without doubt one of the most successful approximations in the history of fluid mechanics. This is certainly true for Newtonian fluids ${ }^{1)}$ but for non-Newtonian fluids, the theory is still regarded as incomplete. ${ }^{2-10)}$ A major obstacle in extending the theory to non-Newtonian fluids is in the diversity of their constitutive behavior meaning that each fluid model should be treated separately. Furthermore, the nonlinearity introduced by their shear-dependent viscosity and/or elasticity often gives rise to a formidable mathematical task which cannot be solved, at times, even numerically. Understandably, the situation becomes much more complicated when the viscosity of the fluid is time-dependent (e.g., when the fluid is thixotropic). Such fluid systems are quite frequent in industrial applications (e.g., drilling muds) with the common effect being that their viscosity drops with the progress of time at any given shear rate. $^{11,12)}$

Due to the complexity of their rheological behavior, working with thixotropic fluids is not an easy task. A major problem is the lack of a robust and easy-to-use rheological model which can describe such behavior. Among different rheological models available to represent such fluid systems

$\dagger$ Corresponding author
Harris model is without doubt one of the simplest ones, albeit admittedly not the best one. ${ }^{13,14)}$ Interestingly, the model developed by Harris can also represent purely-viscous shearthinning fluids for certain set of parameter values. Harris tried this version of his rheological model to study Blasius flow. He relied on the technique of similarity solution and reduced the boundary layer equations into a single ODE. But, the equation so obtained was realized to be too formidable to render itself to an analytical or even numerical solution so that it remained unsolved until recently. As a matter of fact, in a recent work Sadeqi et $\mathrm{al}^{15)}$ relied on a robust numerical method to tackle Blasius flow of shear-thinning fluids obeying Harris model. They also showed that Harris model can represent thixotropic fluids only for certain values of the model parameters.

In the present work we would like to extend the work carried out in Ref. 15 to Sakiadis flow. ${ }^{16)}$ Due to the strong nonlinearity of the governing equation, we have decided to rely on the homotopy analysis method (HAM) in the present work. ${ }^{17)}$ Unlike perturbation techniques, HAM is independent of the smallness/largeness of any parameter involved in the problem. In addition, it provides a simple way to ensure the convergence of the series-solution so that one can always come up with a sufficiently accurate approximation to the solution (even for strongly non-linear problems). Furthermore, unlike all other analytical techniques, the homotopy analysis method provides great freedom in choosing the so-called 
auxiliary linear operator so that one can approximate a nonlinear problem more effectively by means of suitable base functions. It should be noted that the homotopy analysis method has been successfully applied to many nonlinear problems. ${ }^{18-22)}$ It is expected that this robust mathematical method would enable us to investigate the effect of the material parameters in the Harris model on the wall shear stress at any given location along the moving sheet.

The present work is organized as follows. We will start with describing the physical problem and the mathematical formulations for the Harris model in Sakiadis flow. We will then proceed with reducing the governing equations into an ordinary differential equation using a suitable similarity variable. The method of solution (i.e., HAM) is described next. Results are presented together with giving some discussions on their significance. The work is concluded by highlighting its major findings.

\section{MATHEMATICAL FORMULATION}

As mentioned above, the main objective of the present work is to investigate the effects of the parameters appearing in the Harris model on the characteristics of its boundary layer in Sakiadis flow. The flow itself has been depicted schematically in Fig. 1. As can be seen in this figure, a rigid flat plate is being pulled with a constant velocity in an otherwise stationary fluid. The flow is assumed to be occurring under steady and laminar conditions. Part of the interest in this particular flow arises from its technological importance in high-speed production of adhesive tapes. Obviously, the flow is of particular interest from a fundamental point of view. Since the pioneering work of Sakiadis on Newtonian fluids in this particular geometry using boundary layer theory, different aspects of the flow have been studied by several researchers. ${ }^{23-26)}$ This includes non-

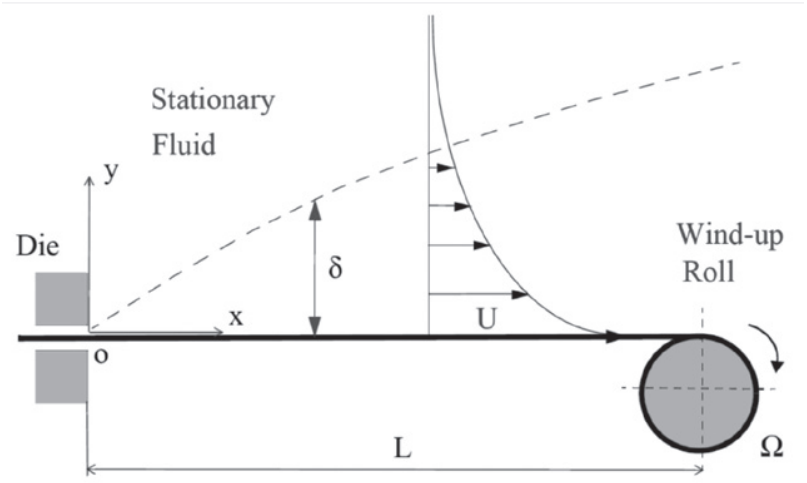

Fig. 1. Schematic showing the flow geometry used for the analysis.
Newtonian fluids too. Surprisingly, however, Harris model has not previously been tried in Sakiadis flow in any study carried out in the past.

To derive the boundary layer equations for an arbitrary fluid, one should start from Cauchy equation of motion together with the continuity equation. For an incompressible fluid undergoing a laminar, two-dimensional flow under isothermal condition, the governing equations are:

$$
\begin{aligned}
& \rho\left(u \frac{\partial u}{\partial x}+v \frac{\partial u}{\partial y}\right)=\frac{\partial \tau_{x x}}{\partial x}+\frac{\partial \tau_{x y}}{\partial y} \\
& \rho\left(u \frac{\partial v}{\partial x}+v \frac{\partial v}{\partial y}\right)=\frac{\partial \tau_{y x}}{\partial x}+\frac{\partial \tau_{y y}}{\partial y} \\
& \frac{\partial u}{\partial x}+\frac{\partial v}{\partial y}=0
\end{aligned}
$$

where it has tacitly been assumed that there is no pressure gradient involved anywhere in the flow.

\subsection{The Harris Rheological Model}

In the present work, we have decided to rely on the Harris model for representing the rheology of the shear-thinning fluid of interest. ${ }^{13,14)}$ This rheological model represents purelyviscous fluids so that we have,

$$
\tau_{i j}=2 \mu\left(I_{2 d}, t\right) d_{i j}
$$

where the viscosity is a function of time. In this equation, $\mathrm{II}_{2 \mathrm{~d}}$ is the second invariant of the deformation-rate tensor and is defined by,

$$
\mathrm{II}_{2 \mathrm{~d}}=\left(2 \mathrm{~d}_{\mathrm{ij}}\right)^{2}=4\left[\left(\frac{\partial \mathrm{u}}{\partial \mathrm{x}}\right)^{2}+\frac{1}{2}\left(\frac{\partial \mathrm{u}}{\partial \mathrm{y}}+\frac{\partial \mathrm{v}}{\partial \mathrm{x}}\right)^{2}+\left(\frac{\partial \mathrm{v}}{\partial \mathrm{y}}\right)^{2}\right]>0
$$

In the Harris model, in its most general form, the viscosity is related to $\mathrm{II}_{2 \mathrm{~d}}$ through the following equation ${ }^{14)}$ :

$$
\mu(t)=\mu_{0}-R_{1} I_{2 d}+R_{2} \frac{D_{2 d}}{D t}
$$

where $\mathrm{D} / \mathrm{Dt}$ is the material derivative, and $\mathrm{R}_{1}$ and $\mathrm{R}_{2}$ are model parameters. In his book ${ }^{13)}$ Harris argues that the model can represent thixotropic fluids on the condition that the model parameters, $\mathrm{R}_{1}$ and/or $\mathrm{R}_{2}$, are allowed to be time-dependent. Since one of the main characteristics of thixotropic fluids is that in simple shear flow (i.e., where $\frac{\mathrm{DII}_{2 \mathrm{~d}}}{\mathrm{Dt}}$ is equal to zero) their viscosity should gradually drop in time (until it reaches an equilibrium value) one can conclude that for the Harris model to represent thixotropic fluids, $\mathrm{R}_{1}$ should definitely be time-dependent while $R_{2}$ can be constant. With $R_{1}$ and $R_{2}$ being both constant, the model can represent shear-thinning 
or shear-thickening fluids only, but not thixotropic fluids. In the present work, we assume that $\mathrm{R}_{1}$ and $\mathrm{R}_{2}$ are both constant as we are primarily interested in the shear-thinning aspect of the Harris model. But, for the Harris model to represent shearthinning fluids the sign and magnitude of $R_{1}$ and $R_{2}$ cannot be arbitrary. For example, for the viscosity of Harris model to drop when shear rate is increased in simple shear (i.e., where $\frac{\mathrm{DII}_{2 \mathrm{~d}}}{\mathrm{Dt}}=0$ ) from Eq. 4 it can be concluded that $\mathrm{R}_{1}$ should definitely be a positive number. As to the sign of $\mathrm{R}_{2}$, it should be noted that in Sakiadis flow, the material derivative $\frac{\mathrm{DII}_{2 \mathrm{~d}}}{\mathrm{Dt}}$ is obviously negative, as can be deduced from Fig. 1. This means that, if $R_{2}$ is taken as a positive number, we would definitely be dealing with a shear-thinning fluid regardless of the magnitude of $R_{1}$. However, even for negative values of $\mathrm{R}_{2}$, Harris model can still represent shear-thinning fluids provided that $R_{1}$ is large enough. Therefore, we will allow $R_{2}$ to take either positive or negative values. With $R_{1}$ and $R_{2}$ being constant, Eq. (4) can be simplified using boundary layer approximation [see Ref. 16 for the details]. The result is:

$$
\mu=\mu_{0}-2 R_{1}\left(\frac{\partial u}{\partial y}\right)^{2}+4 R_{2}\left[u\left(\frac{\partial u}{\partial y} \frac{\partial^{2} u}{\partial x \partial y}\right)+v\left(\frac{\partial u}{\partial y} \frac{\partial^{2} u}{\partial y^{2}}\right)\right]
$$

Now, based on the conventional boundary layer theory, it is quite straightforward to show that the y-momentum equation is completely dropped from the analysis. On the other hand, the $\mathrm{x}$-momentum equation is reduced to [see Ref. 16]:

$$
\begin{aligned}
\rho\left(u \frac{\partial u}{\partial x}\right. & \left.+v \frac{\partial u}{\partial y}\right) \\
& =\mu_{0}\left(\frac{\partial^{2} u}{\partial y^{2}}\right)-6 R_{1}\left(\frac{\partial u}{\partial y}\right)^{2}\left(\frac{\partial^{2} u}{\partial y^{2}}\right) \\
& +4 R_{2}\left[\left(\frac{\partial u}{\partial y}\right)\left(\frac{\partial^{2} u}{\partial y^{2}}\right)\left(u \frac{\partial^{2} u}{\partial x \partial y}+v\left(\frac{\partial^{2} u}{\partial y^{2}}\right)\right)\right. \\
& \left.+\left(\frac{\partial u}{\partial y}\right)^{2}\left(u \frac{\partial^{3} u}{\partial x \partial y^{2}}+v \frac{\partial^{3} u}{\partial y^{3}}+\frac{\partial u}{\partial y} \frac{\partial^{2} u}{\partial x \partial y}+\frac{\partial v}{\partial y} \frac{\partial^{2} u}{\partial y^{2}}\right)\right]
\end{aligned}
$$

This is the boundary layer equation for the Harris fluid in any two-dimensional flow when there is no pressure gradient involved. This equation together with the continuity equation, Eq. (1c), constitute the governing equations for Sakiadis flow of the Harris fluid. As to the boundary conditions required to solve this equation, we rely on the no-slip and no-penetration conditions at the wall. As an extra boundary condition, the velocity profiles are required to merge smoothly with the quiescent fluid resting outside the boundary layer. As an extra boundary condition, we assume that far from the plate (i.e., outside the boundary layer) the fluid is stress-free. Therefore, we have at our disposal the following four physical boundary conditions:

$$
\mathrm{f}(0)=0 ; \quad \mathrm{f}^{\prime}(0)=1 ; \quad \mathrm{f}^{\prime}(\infty)=0 ; \quad \mathrm{f}^{\prime \prime}(\infty)=0
$$

Since the flow is incompressible and two-dimensional, the concept of stream function $\psi(\mathrm{x}, \mathrm{y})$ can be invoked such that we have: $\mathrm{u}=\partial \psi / \partial \mathrm{y}, \mathrm{v}=-\partial \psi / \partial \mathrm{x}$. Now, in a search for a similarity solution, like Harris ${ }^{14)}$ the following similarity variable can be introduced:

$$
\eta(x, y)=y \sqrt{\frac{U_{\infty}}{v_{0} x}}
$$

where $\mathrm{v}_{0}=\mu_{0} / \rho$. With this similarity variable, the stream function can be made dimensionless as,

$$
\mathrm{f}(\eta)=\frac{\psi}{\sqrt{\mathrm{v}_{0} \mathrm{xU}_{\infty}}}
$$

In the terms of the dimensionless stream function, f, Eq. 6 becomes (see Ref. 16 for the details):

$$
\begin{aligned}
& \mathrm{K}_{1}(\mathrm{x}) \mathrm{f}^{\prime{ }^{2}} \mathrm{f}^{\prime \prime \prime}+\mathrm{K}_{2}(\mathrm{x}) \mathrm{f}^{\prime \prime} \\
& {\left[\frac{3}{2} \mathrm{f}^{\prime} \mathrm{f}^{\prime \prime} \mathrm{f}^{\prime \prime \prime}+\frac{1}{2} \mathrm{ff}^{\prime \prime^{2}}+\frac{1}{2} \mathrm{f}^{\prime \prime}\left(\mathrm{ff^{ \prime \prime \prime \prime }}+\mathrm{f}^{\prime^{2}}\right)\right]} \\
& +2 \mathrm{f}^{\prime \prime \prime}+\mathrm{ff}^{\prime \prime}=0
\end{aligned}
$$

where $\mathrm{K}_{1}$ and $\mathrm{K}_{2}$ are dimensionless variables related to $\mathrm{R}_{1}$ and $\mathrm{R}_{2}$ by:

$$
\mathrm{K}_{1}(\mathrm{x})=-12\left(\frac{\mathrm{R}_{1} \mathrm{U}_{\infty}^{3}}{\rho \mathrm{xv}^{2}}\right) ; \quad \mathrm{K}_{2}(\mathrm{x})=-8\left(\frac{\mathrm{R}_{2} \mathrm{U}_{\infty}^{4}}{\rho \mathrm{x}^{2} \mathrm{v}^{2}}\right)
$$

As can be seen from the above relationships, $\mathrm{K}_{1}$ and $\mathrm{K}_{2}$ are both function of $\mathrm{x}$. Therefore, it can be concluded that, unlike power-law fluids for which a self-similar solution does exist, Harris fluid lacks a self-similar solution in Sakiadis flow. Sincce the flow lacks a self-similar solution, therefore we look for a local similarity solution. Such a solution, if it can be found, is still valuable in that it can enable us to investigate the effects of shear-thinning on the velocity profile above the moving plate. Having found the velocity profile, one can then proceed with calculating the wall shear stress as ${ }^{16)}$,

$$
\tau_{\mathrm{w}}=\left.\left.\mu\right|_{\mathrm{y}=0}\left(\frac{\partial \mathrm{u}}{\partial \mathrm{y}}\right)\right|_{\mathrm{y}=0}
$$

where, based on Eq. (5), we have,

$$
\left.\mu\right|_{y=0}=\mu_{0}-2 R_{1}(\partial u / \partial y)^{2}
$$

In terms of "f" the wall shear stress can be written as: 


$$
\begin{aligned}
\tau_{\mathrm{w}} & =\mu_{0} \mathrm{f}^{\prime \prime}(0)-\left(2 \mathrm{R}_{1} \mathrm{U}_{\infty}^{3} / \mathrm{v}_{0} \mathrm{x}\right)\left[\mathrm{f}^{\prime \prime}(0)\right]^{3} \\
& =\mu_{0}\left\{\mathrm{f}^{\prime \prime}(0)+\frac{\mathrm{K}_{1}}{6}\left[\mathrm{f}^{\prime \prime}(0)\right]^{3}\right\}
\end{aligned}
$$

From this equation it can be concluded that at a given $\mathrm{x}$ location, the wall shear stress is affected directly by $R_{1}$ but only indirectly by $\mathrm{R}_{2}$ (i.e., through $\mathrm{f}^{\prime \prime}(0)$ ).

\section{METHOD OF SOLUTION}

In the present work we try to find an analytical solution for Eq. (10) albeit admittedly it looks too formidable. In order to investigate separate effects of $R_{1}$ and $R_{2}$ on the velocity profile and wall shear stress at a given location along the plate, we focus on the two asymptotic cases of $\mathrm{K}_{1}=0$ and $\mathrm{K}_{2}=0$. Due to strong non-linear nature of Eq. (10), we rely on the homotopy analysis method (HAM) for this purpose.

\subsection{Zeroth-Order Deformation Equation}

Liao $^{17)}$ has developed an efficient analytical method for solving highly non-linear differential equations named the homotopy analysis method. Following Liao, we express $\mathrm{f}(\eta)$ by a set of base functions as:

$$
\left\{\eta, \eta^{n} e^{-m \beta \eta} \mid n \geq 0, m \geq 1, \beta>0\right\}
$$

where we have,

$$
f(\eta)=\eta+\sum_{m=1}^{+\infty} \sum_{n=0}^{+\infty} a_{m, n} \eta^{n} e^{-m \beta \eta}
$$

with $\mathrm{a}_{\mathrm{m}, \mathrm{n}}$ being coefficients and $\beta>0$ being a spatial - scale parameter. This provides us with the so-called rule-of-solution expression for $\mathrm{f}(\eta)$. According to this rule we choose:

$$
\mathrm{f}_{0}(\eta)=\frac{1}{\beta}-\frac{1}{\beta} \mathrm{e}^{-\beta \eta}
$$

As the initial guess for $f(\eta)$ we set:

$$
\mathcal{L}[\Phi(\eta ; q)]=\frac{\partial^{3} \Phi(\eta ; q)}{\partial \eta^{3}}-\beta^{2} \frac{\partial \Phi(\eta ; q)}{\partial \eta}
$$

and

$$
\mathcal{L}[\Phi(\eta ; q)]=\frac{\partial^{4} \Phi(\eta ; q)}{\partial \eta^{4}}-\beta^{2} \frac{\partial \Phi(\eta ; q)}{\partial \eta}
$$

respectively for $\mathrm{K}_{2}=0$ and $\mathrm{K}_{1}=0$. We then introduce the auxiliary linear operator, which has the following properties:

$$
\mathcal{L}\left[\mathrm{C}_{1} \mathrm{e}^{\beta \eta}+\mathrm{C}_{2} \mathrm{e}^{-\beta \eta}+\mathrm{C}_{3}\right]=0
$$

and

$$
\mathcal{L}\left[\mathrm{C}_{1} \mathrm{e}^{\beta \eta}+\mathrm{C}_{2} \mathrm{e}^{-\beta \eta}+\mathrm{C}_{3}+\eta \mathrm{C}_{4}\right]=0
$$

respectively for $\mathrm{K}_{2}=0$ and $\mathrm{K}_{1}=0$, where $\mathrm{C}_{1}, \mathrm{C}_{2}, \mathrm{C}_{3}$ and $\mathrm{C}_{4}$ are constants. Based on Eq. (10), we are led to define the following nonlinear operators:

$$
\begin{aligned}
\mathcal{N}[\Phi(\eta ; q)]= & K_{1}\left(\frac{\partial^{2} \Phi(\eta ; q)}{\partial \eta^{2}}\right)^{2}\left(\frac{\partial^{3} \Phi(\eta ; q)}{\partial \eta^{3}}\right) \\
& +2 \frac{\partial^{3} \Phi(\eta ; q)}{\partial \eta^{3}}+\frac{\partial \Phi(\eta ; q)}{\partial \eta} \frac{\partial^{2} \Phi(\eta ; q)}{\partial \eta^{2}}
\end{aligned}
$$

and

$$
\begin{aligned}
& \mathcal{N}[\Phi(\eta ; q)] \\
& =K_{2} \frac{\partial^{2} \Phi(\eta ; q)}{\partial \eta^{2}}\left[\frac{3}{2}\left(\frac{\partial \Phi(\eta ; q)}{\partial \eta}\right)\left(\frac{\partial^{2} \Phi(\eta ; q)}{\partial \eta^{2}}\right)\left(\frac{\partial^{3} \Phi(\eta ; q)}{\partial \eta^{3}}\right)\right. \\
& +\frac{1}{2}\left(\frac{\partial \Phi(\eta ; q)}{\partial \eta}\right)\left(\frac{\partial^{3} \Phi(\eta ; q)}{\partial \eta^{3}}\right)^{2} \\
& \left.+\frac{1}{2} \frac{\partial^{2} \Phi(\eta ; q)}{\partial \eta^{2}}\left(\left(\frac{\partial \Phi(\eta ; q)}{\partial \eta}\right)\left(\frac{\partial^{4} \Phi(\eta ; q)}{\partial \eta^{4}}\right)+\left(\frac{\partial^{2} \Phi(\eta ; q)}{\partial \eta^{2}}\right)^{2}\right)\right] \\
& +2 \frac{\partial^{3} \Phi(\eta ; q)}{\partial \eta^{3}}+\frac{\partial \Phi(\eta ; q)}{\partial \eta} \frac{\partial^{2} \Phi(\eta ; q)}{\partial \eta^{2}}
\end{aligned}
$$

respectively for $\mathrm{K}_{2}=0$ and $\mathrm{K}_{1}=0$. Now we construct the socalled zeroth-order equation as:

$$
(1-\mathrm{q}) \mathcal{L}\left[\Phi(\eta ; \mathrm{q})-\mathrm{f}_{0}(\eta)\right]=\mathrm{q} \hbar \mathcal{N}[\Phi(\eta ; \mathrm{q})]
$$

where $\mathrm{h}$ is an auxiliary parameter subject to the following boundary conditions:

$$
\Phi(0 ; q)=0,\left.\frac{\partial \Phi(\eta ; q)}{\partial \eta}\right|_{\eta=0}=1,\left.\frac{\partial \Phi(\eta ; q)}{\partial \eta}\right|_{\eta=+\infty}=0
$$

and

$$
\begin{aligned}
\Phi(0 ; q)=0,\left.\frac{\partial \Phi(\eta ; q)}{\partial \eta}\right|_{\eta=0}=1, \\
\left.\frac{\partial \Phi(\eta ; q)}{\partial \eta}\right|_{\eta=+\infty}=0,\left.\quad \frac{\partial^{2} \Phi(\eta ; q)}{\partial \eta^{2}}\right|_{\eta=+\infty}=0
\end{aligned}
$$

where $\mathrm{q} \in[0,1]$ is an embedding parameter. Obviously, when $\mathrm{q}=0$ and $\mathrm{q}=1$, the above zeroth-order deformation have the solutions:

$$
\Phi(\eta ; 0)=\mathrm{f}_{0}(\eta)
$$

and

$$
\Phi(\eta ; 1)=f(\eta)
$$

respectively. Thus as $\mathrm{q}$ increases from 0 to $1, \Phi(\eta ; q)$ varies from the initial guess $f_{0}(\eta)$ to the solution $f(\eta)$. So, expanding 
$\Phi(\eta ; q)$ in a Taylor's series with respect to the embedding parameter q, we have:

$$
\Phi(\eta ; q)=\Phi(\eta ; 0)+\sum_{\mathrm{k}=1}^{+\infty} \mathrm{f}_{\mathrm{k}}(\eta) \mathrm{q}^{\mathrm{k}}
$$

where

$$
\mathrm{f}_{\mathrm{k}}(\eta)=\left.\frac{1}{\mathrm{k} !} \frac{\partial^{\mathrm{k}} \Phi(\eta ; \mathrm{q})}{\partial \mathrm{q}^{\mathrm{k}}}\right|_{\mathrm{q}=0}
$$

Note that the above equation contains the auxiliary parameter. Assuming that it is properly chosen so that the proposed series is convergent at $\mathrm{q}=1$, we obtain:

$$
f(\eta)=f_{0}(\eta)+\sum_{k=1}^{+\infty} f_{k}(\eta)
$$

\subsection{High-Order Deformation Equation}

For simplicity we define the vector:

$$
\overrightarrow{\mathrm{f}}_{\mathrm{k}}=\left\{\mathrm{f}_{0}, \mathrm{f}_{1}, \mathrm{f}_{2}, \ldots, \mathrm{f}_{\mathrm{k}}\right\}
$$

Differentiating the zeroth-order deformation equation $\mathrm{k}$ times with respect to $\mathrm{q}$, then setting $\mathrm{q}=0$, and finally dividing them by $\mathrm{k}$ !, we obtain the $\mathrm{k}^{\text {th }}$-order deformation equations as:

$$
\mathcal{L}\left[f_{k}(\eta)-\chi_{k} f_{k-1}(\eta)\right]=h R_{k}\left(f_{k-1}\right)
$$

subject to the boundary conditions:

$$
\mathrm{f}_{\mathrm{k}}(0)=\mathrm{f}_{\mathrm{k}}^{\prime}(0)=\mathrm{f}_{\mathrm{k}}^{\prime}(+\infty)=0
$$

And

$$
\mathrm{f}_{\mathrm{k}}(0)=\mathrm{f}_{\mathrm{k}}^{\prime}(0)=\mathrm{f}_{\mathrm{k}}^{\prime}(+\infty)=\mathrm{f}_{\mathrm{k}}^{\prime \prime}(+\infty)=0
$$

where,

$$
\mathrm{R}_{\mathrm{k}}\left(\overrightarrow{\mathrm{f}}_{\mathrm{k}-1}\right)=\left.\frac{1}{(\mathrm{k}-1) !} \frac{\partial^{\mathrm{k}-1} \mathcal{N}[\Phi(\eta ; \mathrm{q})]}{\partial \mathrm{q}^{\mathrm{k}-1}}\right|_{\mathrm{q}=0}
$$

and

$$
\chi_{\mathrm{m}}=\left\{\begin{array}{l}
0, \mathrm{~m}=1 \\
1, \mathrm{~m}>1 .
\end{array}\right.
$$

Note that $R_{k}\left(\overrightarrow{(}_{k-1}\right)$ depends on $K_{1}=0$ or $K_{2}=0$. For $K_{2}=0$ we have:

$$
\begin{aligned}
\mathrm{R}_{\mathrm{k}}\left(\overrightarrow{\mathrm{f}}_{\mathrm{k}-1}\right)= & \mathrm{K}_{1}\left[\sum_{\mathrm{i}=0}^{\mathrm{k}-1} \mathrm{f}_{\mathrm{i}}^{\prime \prime \prime} \sum_{\mathrm{j}=0}^{\mathrm{k}-1-\mathrm{i}} \mathrm{f}_{\mathrm{k}-1-\mathrm{i}-\mathrm{j}}^{\prime \prime} \mathrm{f}_{\mathrm{j}}^{\prime \prime}\right] \\
& +\left[\sum_{\mathrm{i}=0}^{\mathrm{k}-1} \mathrm{f}_{\mathrm{i}} \mathrm{f}_{\mathrm{k}-1-\mathrm{i}}^{\prime \prime}\right]+2 \mathrm{f}_{\mathrm{k}-1}^{\prime \prime}
\end{aligned}
$$

and for $K_{1}=0$ we have:

$$
\begin{aligned}
\mathrm{R}_{\mathrm{k}}\left(\mathrm{f}_{\mathrm{k}-1}\right)= & \mathrm{K}_{2}\left[\sum _ { \mathrm { i } = 0 } ^ { \mathrm { k } - 1 } \mathrm { f } _ { \mathrm { k } - 1 - \mathrm { i } } ^ { \prime \prime } \left(\left(\frac{3}{2} \sum_{\mathrm{j}=0}^{\mathrm{i}} \mathrm{f}_{\mathrm{j}}^{\prime \prime \prime} \sum_{\mathrm{l}=0}^{\mathrm{i}-\mathrm{j}} \mathrm{f}_{\mathrm{i}-\mathrm{j}-1}^{\prime} \mathrm{f}_{\mathrm{l}}^{\prime \prime \prime}\right)\right.\right. \\
& +\left(\frac{1}{2} \sum_{\mathrm{m}=0}^{\mathrm{i}} \mathrm{f}_{\mathrm{i}-\mathrm{m}} \sum_{\mathrm{n}=0}^{\mathrm{m}} \mathrm{f}_{\mathrm{m}-\mathrm{n}}^{\prime \prime \prime} \mathrm{f}_{\mathrm{n}}^{\prime \prime \prime}\right) \\
& \left.\left.+\left(\frac{1}{2} \sum_{\mathrm{P}=0}^{\mathrm{i}} \mathrm{f}_{\mathrm{i}-\mathrm{p}}^{\prime \prime} \sum_{\mathrm{q}=0}^{\mathrm{p}} \mathrm{f}_{\mathrm{p}-\mathrm{q}} \mathrm{f}_{\mathrm{q}}^{\prime \prime \prime \prime}+\mathrm{f}_{\mathrm{p}-\mathrm{q}}^{\prime \prime} \mathrm{f}_{\mathrm{q}}^{\prime \prime}\right)\right\}\right] \\
& +\left[\sum_{\mathrm{i}=0}^{\mathrm{k}-1} \mathrm{f}_{\mathrm{i}} \mathrm{f}_{\mathrm{k}-1-\mathrm{i}}^{\prime \prime}\right]+2 \mathrm{f}_{\mathrm{k}-1}^{\prime \prime}
\end{aligned}
$$

Let $f_{k}^{*}(\eta)$ denote the particular solution of Eqs. (29) and (30). Using Eqs. (17) and (18) the general solutions for $K_{2}=0$ becomes:

$$
f_{k}(\eta)=f_{k}^{*}(\eta)+C_{1} e^{\beta \eta}+C_{2} e^{-\beta \eta}+C_{3}
$$

where the coefficients $\mathrm{C}_{1}, \mathrm{C}_{2}$ and $\mathrm{C}_{3}$ are determined by the boundary conditions, that is:

$$
\mathrm{C}_{1}=0, \quad \mathrm{C}_{2}=\left.\frac{1}{\beta} \frac{\partial \mathrm{f}_{\mathrm{k}}^{*}}{\partial \eta}\right|_{\eta=0}, \quad \mathrm{C}_{3}=-\mathrm{C}_{2}-\mathrm{f}_{\mathrm{k}}^{*}(0)
$$

and for $K_{1}=0$ :

$$
f_{k}(\eta)=f_{k}^{*}(\eta)+C_{1} e^{\beta \eta}+C_{2} e^{-\beta \eta}+C_{3}+\eta C_{4}
$$

where the coefficients $\mathrm{C}_{1}, \mathrm{C}_{2}, \mathrm{C}_{3}$ and $\mathrm{C}_{4}$ are determined by the boundary conditions:

$$
\begin{aligned}
& C_{1}=0, \quad C_{4}=-\left.\frac{\partial f_{k}^{*}}{\partial \eta}\right|_{\eta=+\infty}, \\
& C_{2}=\frac{1}{\beta} C_{4}+\left.\frac{1}{\beta} \frac{\partial f_{k}^{*}}{\partial \eta}\right|_{\eta=0}, \quad C_{3}=-C_{2}-f_{k}^{*}(0)
\end{aligned}
$$

In this way, we solve the linear Eqs. (29) and (30) one after the other (in the order of $\mathrm{k}=1,2,3$, etc) for $\mathrm{K}_{1}=0$ and $\mathrm{K}_{2}=0$ by means of the symbolic computation software Mathematica. As long as the series-solution is convergent it should converge to one the solutions of the original equation. Obviously, it is important to ensure the convergence of the solution series for all possible variables $0 \leq \mathrm{x}<+\infty$ and $0 \leq \mathrm{y}<+\infty$, otherwise, the series-solution is useless. Fortunately, our series-solution contains the convergence-control parameter and also the spatial-scale parameter [see Ref. 16] which provides us with a simple way to adjust and control the convergence region and the rate of convergence of the series solution, as mentioned by Liao. $^{17)}$ 
Using the technique described above, we have been able to obtain converged results for Harris model parameters in the range of $-4<\mathrm{K}_{1}<0$ and $-2<\mathrm{K}_{2}<+1$. For the $16^{\text {th }}$-order solution, the series contained roughly 30,000 terms. In the Appendix, we have shown the $3^{\text {rd }}$-order series-solution which shows how quickly the number of terms in the series can rise by increasing the order of the solution.

\section{RESULTS AND DISCUSSION}

The code developed in the present work had to be verified first and this was easily done by comparing its output for the case of $R_{1}=R_{2}=0$ (i.e. the Newtonian case) with published data in Ref. 1. Figure 2 shows a comparison between the results obtained in this work (using HAM) with those reported in Ref. 1 for Sakiadis flow of Newtonian fluids. ${ }^{1)}$ As can be seen in this figure, the two sets data are virtually the same demonstrating that the code developed in this work is working properly.

Having verified the code, it was then used to investigate the separate effects of $K_{1}$ and $K_{2}$ on the velocity profile and wall shear stress at a given location above the plate.

\subsection{Effect of $\mathrm{K}_{1}$}

Figure 3 shows the results obtained for the velocity profiles, $\mathrm{f}^{\prime}$, as a function of $\mathrm{K}_{1}$ for the case of $\mathrm{K}_{2}=0$. This figure also includes the Newtonian case for comparison purposes. As mentioned above, $R_{1}$ is a positive number which means that $\mathrm{K}_{1}$ is a negative number (see Eq. 11). As can be seen in Fig. 3 , by an increase in $\mathrm{K}_{1}$ fluid velocity is decreased at any given location above the plate. That is to say that, the magnitude of the material constant $R_{1}$ in the Harris model has a retarding effect on the velocity imparted to the fluid above the sheet.

Figure 4 shows the effect of $\mathrm{K}_{1}$ on $\mathrm{f}^{\prime \prime}$ profile for the case of

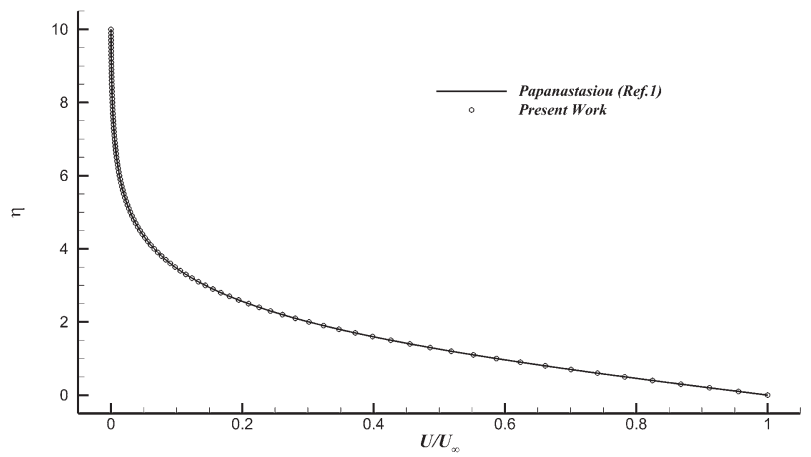

Fig. 2. A comparison between our homotopy results and the numerical results reported in Ref. 1 for Sakiadis flow of Newtonian fluids.
$\mathrm{K}_{2}=0$ (obtained when $\hbar=-0.5$, and $\beta=+0.5$ ). As can be seen in this figure, the effect of $\mathrm{K}_{1}$ on $\mathrm{f}^{\prime \prime}$ is non-monotonic. Of particular importance is the magnitude of $\mathrm{f}^{\prime \prime}(0)$ at the wall as it is related to the wall shear stress through Eq. 14. As can be seen in Fig. 4, $\mathrm{f}^{\prime \prime}(0)$ is increased by an increase in $\mathrm{K}_{1}$. Thus, an increase in the wall shear is expected to arise when $\mathrm{K}_{1}$ is increased. Figure 5 shows that this is indeed the case. Still, this prediction looks puzzling at first sight because an increase in $\mathrm{K}_{1}$ (for $\mathrm{K}_{2}=0$ ) is tantamount to saying that the viscosity of the fluid decreases by an increase in the shear rate. This should be contrasted with the previous finding that for fluids obeying the power-law model shear-thinning gives rise to a decrease in the wall shear stress [see Ref. 27]. Interestingly, a drop in viscosity in the Harris model (as $K_{1}$ is increased) is more than counterbalanced by an increase in the wall shear rate as caused by an overall alteration of the velocity profile (see Eq. 12).

\subsection{Effect of $\mathrm{K}_{2}$}

Figure 6 shows the results obtained for the velocity profiles, $\mathrm{f}^{\prime}$, as a function of $\mathrm{K}_{2}$ for the case of $\mathrm{K}_{1}=0$ (obtained

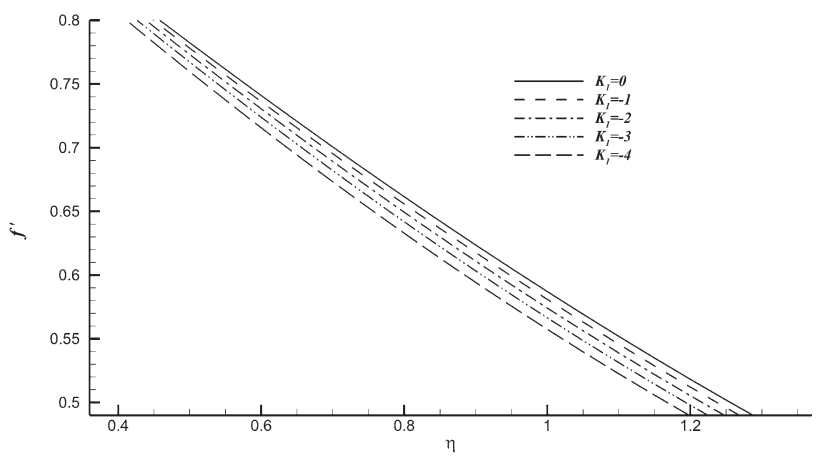

Fig. 3. Effect of $\mathrm{K}_{1}$ on $\mathrm{f}^{\prime}$ for the case of $\mathrm{K}_{2}=0$.

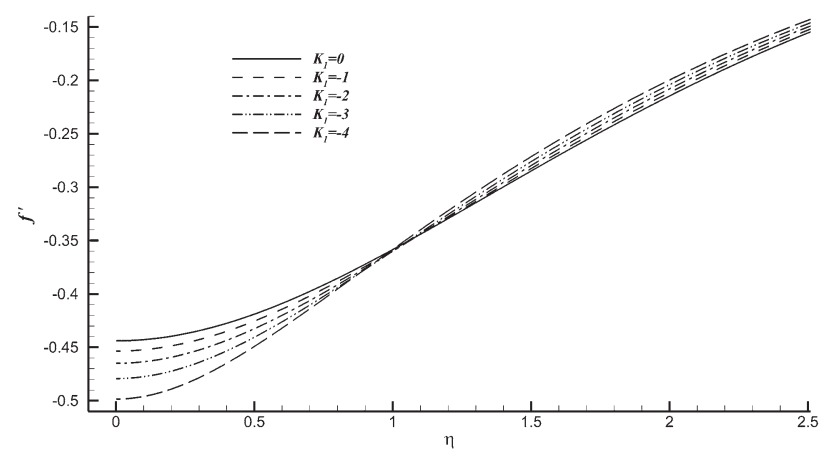

Fig. 4. Effect of $\mathrm{K}_{1}$ on $\mathrm{f}^{\prime \prime}$ for the case of $\mathrm{K}_{2}=0$. 
when $\hbar=-1.8$, and $\beta=+0.65$ ). This figure also includes the Newtonian case for comparison purposes. From Fig. 6 it can be concluded that the sign of $\mathrm{K}_{2}$ has a strong effect on the velocity profile. That is, for $\mathrm{K}_{2}>0$ velocity is increased at any given location above the plate, but for $\mathrm{K}_{2}<0$ its effect is reversed.

Figure 7 shows the effect of $\mathrm{K}_{2}$ on $\mathrm{f}^{\prime \prime}$ for the case of $\mathrm{K}_{1}=0$. As can be seen in this figure. The effect of $\mathrm{K}_{2}$ on $\mathrm{f}^{\prime \prime}$ is nonmonotonic regardless of its sign. From this figure, we can obtain $\mathrm{f}^{\prime \prime}(0)$ which provides us with the wall shear stress through the relationship $\tau_{\mathrm{w}}=\mu_{0} \mathrm{f}^{\prime \prime}(0)$ based on Eq. 14. As can be seen in Fig. 8, the wall shear stress is decreased by an increase in $R_{2}$ provided it is negative. For positive $R_{2}$ an increase in its absolute value gives rise to an increase in the absolute value of the wall shear stress. Since shear-thinning is known to lower the force required to pull the sheet ${ }^{27)}$ one can conclude that $\mathrm{R}_{2}$ must definitely be negative. A comparison between Fig. 5 and Fig. 8 reveals that the effect of $R_{2}$ on the wall shear stress is stronger than the effect of $R_{1}$. Therefore, one can conclude that for the Harris model to comply with experimental results, not only $\mathrm{R}_{2}$ must be negative but its absolute value should also be larger than $R_{1}$.

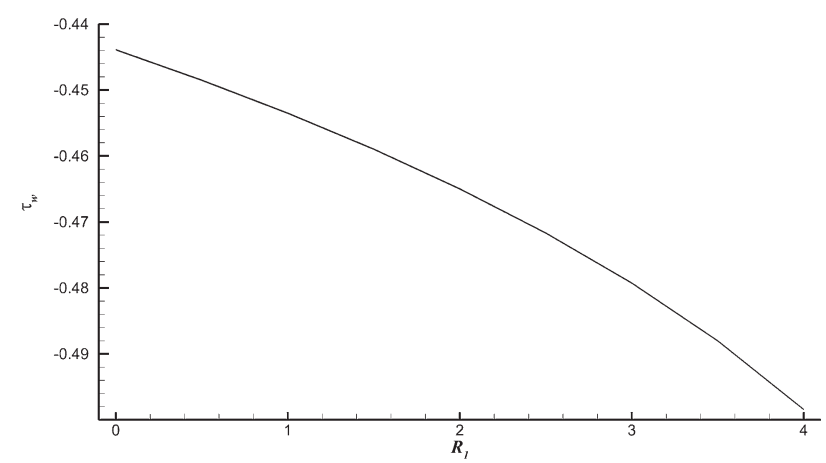

Fig. 5. Effect of $R_{1}$ on the wall shear stress for the case of $R_{2}=0$.

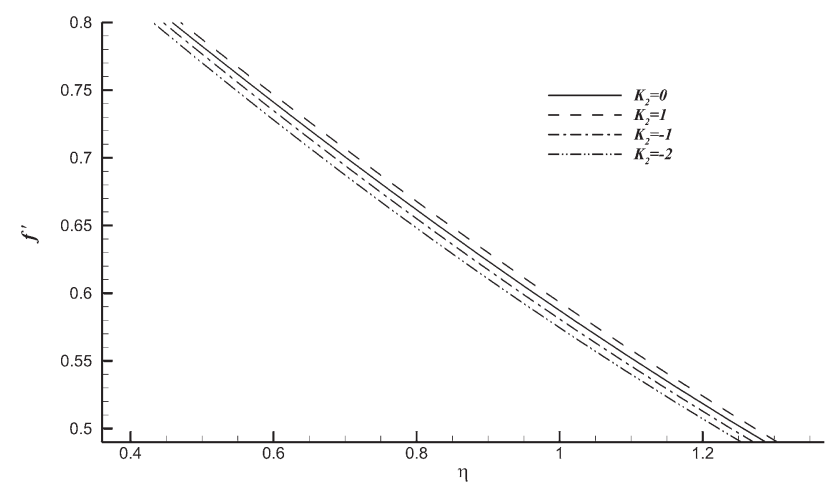

Fig. 6. Effect of $\mathrm{K}_{2}$ on $\mathrm{f}^{\prime}$ for the case of $\mathrm{K}_{1}=0$.

\section{CONCLUDING REMARKS}

In the present work, Sakiadis flow of a Harris fluid is investigated using homotopy analysis method (HAM). This simple fluid model incorporates two material properties to represent a fluid's thixotropic and/or shear-thinning behavior. In this work, we have dealt with the shear-thinning version of the fluid model only, in which the two model parameters are both constant. Based on the results obtained in this work, it can be concluded that for this fluid model to truly represent shear-thinning fluids, the sign and magnitude of the model parameters cannot be arbitrary. As a matter of fact, our results suggest that for shear-thinning behavior to have a diminishing effect on the force required to pull the sheet in Sakiadis flow, $\mathrm{R}_{1}$ should be positive while $\mathrm{R}_{2}$ should be negative. Also, the absolute value of $R_{2}$ should be larger than $R_{1}$ (say, by an order of magnitude). Therefore, for those researchers who intend to rely on this tricky rheological model for investigating the role played by a fluid's shear-thinning behavior on any given fluid mechanics problem, the sign and magnitude of $R_{1}$ and $R_{2}$ should be set properly.

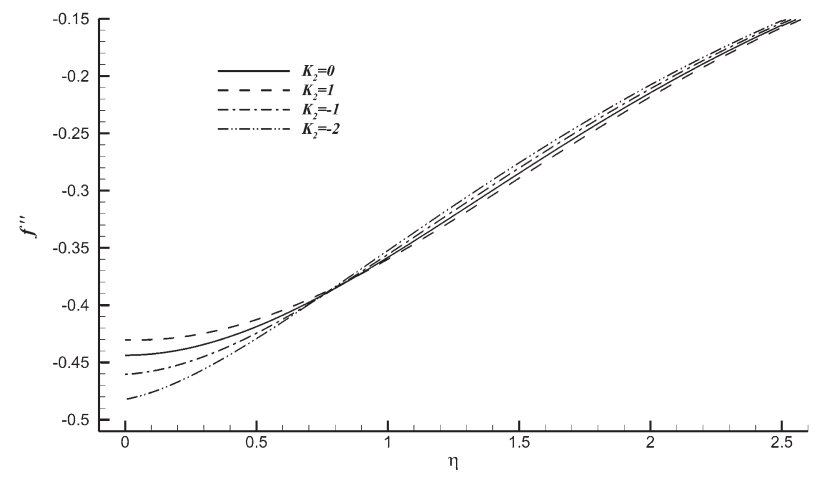

Fig. 7. Effect of $\mathrm{K}_{2}$ on $\mathrm{f}^{\prime \prime}$ for the case of $\mathrm{K}_{1}=0$.

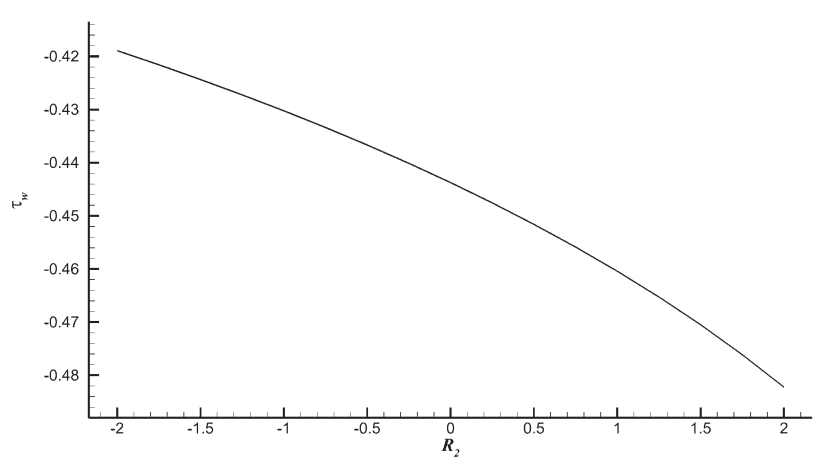

Fig. 8. Effect of $R_{2}$ on the wall shear stress for the case of $\mathrm{R}_{1}=0$. 


\section{ACKNOWLEDGEMENT}

The authors wish to express their thanks to Iran National Science Foundation (INSF) for supporting this work under contract number 92011995. Special thanks are also due to the respectful reviewer(s) for their contributing comments.

\section{REFERENCES}

1) Papanastasiou CT, Georgiou CG, Alexandrou NA, Viscous fluid flow, CRC Press. Boca Raton. London. New York. Washington D.C., 2000.

2) Acrivos A, Shah MJ, Peterson EE, Momentum and heat transfer in laminar boundary layer flow of non-Newtonian fluids past external surfaces, AlChE J, 6, 312 (1960).

3) Beard DW, Walters K, Elastico-viscous boundary layer flow, Proc Camb Phil Soc, 60, 667 (1964).

4) Denn MM, Process fluid mechanics, New Jersey: PrenticeHall Inc, 1980.

5) Rajagopal K, Gupta AS, Wineman AS, On a boundary layer theory for non-Newtonian fluids, Lett Appl Eng Sci, 18, 875 (1980).

6) Andersson HI, Toften TH, Numerical solution of the laminar boundary layer equations for power-law fluids, $J$ NonNewtonian Fluid Mech, 32, 175 (1989).

7) Pop I, Gorla RS, Effect of variable viscosity on flow and heat transfer to a continuous moving flat plate, Int $J$ Sci, 28, 312 (1990).

8) Renardy M, High Weissenberg number boundary layers for the upper-convected Maxwell fluid, J Non-Newtonian Fluid Mech, 68, 125 (1997).

9) Rajagopal KR, Gupta AS, Na TY, Note on the Falkner-Skan flows of a non-Newtonian fluid, $J$ Non-Linear Mech, 18, 313 (1983).

10) Wapperom $P$, Renardy M, Numerical prediction of the boundary layers in the flow around a cylinder using a fixed velocity field, J Non-Newtonian Fluid Mech, 125, 35 (2005).

11) Mewis J, Thixotropy - a general review, J Non-Newtonian Fluid Mech, 6, 1 (1979).

12) Barnes HA, Thixotropy - a review, J Non-Newtonian Fluid Mech, 70, 1 (1997).

13) Harris J, A continuum theory of time-dependent inelastic flow, Rheol Acta, 6, 6 (1967).

14) Harris J, Rheology and non-Newtonian flow, London: Longman, 1977.

15) Sadeqi S, Khabazi N, Sadeghy K, Blasius flow of thixotropic fluids: A numerical study, Commun Nonlinear Sci Numer Simulat, 16, 711 (2011).
16) Arian M, Sakiadis flow of Harris fluids using homotopy method, MSc Thesis, University of Tehran, 2011.

17) Liao SJ, Beyond Purturbation: introduction to the homotopy analysis method, Boca Raton: Chapman \& Hall/CRC Press, 2003

18) Liao SJ, On the analytic solution of magnetohydrodynamic flows of non-Newtonian fluids over a stretching sheet, J Fluid Mech, 488, 189 (2003).

19) Liao SJ, Series solution of unsteady boundary-layer flows over a stretching flat plate, Stud Appl Math, 117, 2529 (2006).

20) Liao SJ, Tan Y, A general approach to obtain series solution of nonlinear differential equations, Stud Appl Math, 119, 297 (2007).

21) Liao SJ, Notes on the homotopy analysis method: Some definitions and theorems, Commun Nonlinear Sci Numer Simulat, 14, 983 (2009).

22) Liao SJ, Pop I, Explicit analytic solution for similarity boundary layer equations, Int $J$ Heat Mass Transfer, 47, 75 (2004).

23) Sakiadis B, Boundary layer behavior on continuous solid surfaces: Boundary layer equations for two-dimensional and axisymmetric flows, AIChE J, 7, 26 (1961).

24) Sakiadis B, Boundary layer behavior on continuous solid surface: The boundary layer on a continuous flat surface, AIChE J, 7, 221 (1961).

25) Tsou F, Sparrow E, Goldstein R, Flow and heat transfer in the boundary layer on a continuous moving surface, Int $J$ Heat Mass Transfer, 10, 219 (1967).

26) Fox VG, Erickson LE, Fan LT, The laminar boundary layer on a moving continuous sheet immersed in a non-Newtonian fluid, AIChE J, 15, 327 (1969).

27) Hassanien IA, Flow and heat transfer on a continuous surface moving in a parallel free stream of power-law fluid, Appl Modelling, 20, 779 (1996).

\section{APPENDIX: The Third-Order Series-Solution}

As mentioned in the main text, we have relied on the Mathematica software to solve symbolically for $\mathrm{f}(\mathrm{h})$. We have been able to obtain result up to a order of 30 , for which the number of terms comprising the series turned out to be more than 30,000. Below, we have shown the results for a thirdorder solution just as a guide. 
$f(\eta)=\frac{113 h^{3}}{17280 \beta^{7}}-\frac{11 e^{-4 \beta \eta} h^{3}}{11520 \beta^{7}}-\frac{55 e^{-3 \beta \eta} h^{3}}{13824 \beta^{7}}-\frac{e^{-2 \beta \eta} h^{3}}{576 \beta^{7}}+\frac{e^{-\beta \eta} h^{3}}{7680 \beta^{7}}+\frac{5 h^{2}}{48 \beta^{5}}$

$-\frac{5 e^{-3 \beta \eta} h^{2}}{192 \beta^{5}}-\frac{e^{-2 \beta \eta} h^{2}}{16 \beta^{5}}-\frac{e^{-\beta \eta} h^{2}}{64 \beta^{5}}-\frac{5 h^{3}}{288 \beta^{5}}+\frac{5 e^{-3 \beta \eta} h^{3}}{1152 \beta^{5}}+\frac{e^{-2 \beta \eta} h^{3}}{96 \beta^{5}}$

$+\frac{e^{-\beta \eta} h^{3}}{384 \beta^{5}}+\frac{h}{2 \beta^{3}}-\frac{e^{-2 \beta \eta} h}{4 \beta^{3}}-\frac{e^{-\beta \eta} h}{4 \beta^{3}}-\frac{h^{2}}{4 \beta^{3}}+\frac{e^{-2 \beta \eta} h^{2}}{8 \beta^{3}}+\frac{e^{-\beta \eta} h^{2}}{8 \beta^{3}}$

$-\frac{h^{3}}{48 \beta^{3}}+\frac{e^{-2 \beta \eta} h^{3}}{96 \beta^{3}}+\frac{e^{-\beta \eta} h^{3}}{96 \beta^{3}}-\frac{203 h^{3} K 1}{172800 \beta^{3}}-\frac{3419 e^{-5 \beta \eta} h^{3} K 1}{691200 \beta^{3}}$

$+\frac{1003 e^{-4 \beta \eta} h^{3} K 1}{115200 \beta^{3}}-\frac{19 e^{-3 \beta \eta} h^{3} K 1}{9216 \beta^{3}}-\frac{e^{-2 \beta \eta} h^{3} K 1}{3840 \beta^{3}}-\frac{91 e^{-\beta \eta} h^{3} K 1}{345600 \beta^{3}}$

$+\frac{1}{\beta}-\frac{e^{-\beta \eta}}{\beta}-\frac{3 h}{2 \beta}+\frac{3 e^{-\beta \eta} h}{2 \beta}-\frac{3 h^{2}}{8 \beta}+\frac{3 e^{-\beta \eta} h^{2}}{8 \beta}-\frac{h^{3}}{16 \beta}+\frac{e^{-\beta \eta} h^{3}}{16 \beta}$

$-\frac{h^{2} \mathrm{~K} 1}{160 \beta}-\frac{37 \mathrm{e}^{-4 \beta \eta} \mathrm{h}^{2} \mathrm{~K} 1}{960 \beta}+\frac{7 \mathrm{e}^{-3 \beta \eta} \mathrm{h}^{2} \mathrm{~K} 1}{128 \beta}+\frac{\mathrm{e}^{-2 \beta \eta} \mathrm{h}^{2} \mathrm{~K} 1}{32 \beta}$

$-\frac{79 \mathrm{e}^{-\beta \eta} \mathrm{h}^{2} \mathrm{~K} 1}{1920 \beta}-\frac{\mathrm{h}^{3} \mathrm{~K} 1}{320 \beta}-\frac{37 \mathrm{e}^{-4 \beta \eta} \mathrm{h}^{3} \mathrm{~K} 1}{1920 \beta}+\frac{7 \mathrm{e}^{-3 \beta \eta} \mathrm{h}^{3} \mathrm{~K} 1}{256 \beta}$

$+\frac{\mathrm{e}^{-2 \beta \eta} \mathrm{h}^{3} \mathrm{~K} 1}{64 \beta}-\frac{79 \mathrm{e}^{-\beta \eta} \mathrm{h}^{3} \mathrm{~K} 1}{3840 \beta}-\frac{\mathrm{hK1} \beta}{8}-\frac{1}{16} \mathrm{e}^{-3 \beta \eta} \mathrm{hK1} \beta$

$+\frac{3}{16} e^{-\beta \eta} h K 1 \beta-\frac{3}{16} h^{2} K 1 \beta-\frac{3}{32} e^{-3 \beta \eta} h^{2} K 1 \beta+\frac{9}{32} e^{-\beta \eta} h^{2} K 1 \beta$

$-\frac{5}{64} h^{3} K 1 \beta-\frac{5}{128} e^{-3 \beta \eta} h^{3} K 1 \beta+\frac{15}{128} e^{-\beta \eta} h^{3} K 1 \beta+\frac{17 h^{3} K 1^{2} \beta}{26880}$

$-\frac{8021 \mathrm{e}^{-6 \beta \eta} \mathrm{h}^{3} \mathrm{~K} 1^{2} \beta}{1612800}+\frac{43 \mathrm{e}^{-5 \beta \eta} \mathrm{h}^{3} \mathrm{~K} 1^{2} \beta}{6144}+\frac{37 \mathrm{e}^{-4 \beta \eta} \mathrm{h}^{3} \mathrm{~K} 1^{2} \beta}{11520}$

$-\frac{257 \mathrm{e}^{-3 \beta \eta} \mathrm{h}^{3} \mathrm{~K} 1^{2} \beta}{46080}+\frac{\mathrm{e}^{-2 \beta \eta} \mathrm{h}^{3} \mathrm{~K} 1^{2} \beta}{1024}-\frac{4093 \mathrm{e}^{-\beta \eta} \mathrm{h}^{3} \mathrm{~K} 1^{2} \beta}{3225600}$

$-\frac{3}{128} h^{2} K 1^{2} \beta^{3}-\frac{3}{256} e^{-5 \beta \eta} h^{2} K 1^{2} \beta^{3}+\frac{3}{256} e^{-3 \beta \eta} h^{2} K 1^{2} \beta^{3}$

$+\frac{3}{128} e^{-\beta \eta} h^{2} K 1^{2} \beta^{3}-\frac{7}{256} h^{3} K 1^{2} \beta^{3}-\frac{7}{512} e^{-5 \beta \eta} h^{3} K 1^{2} \beta^{3}$

$+\frac{7}{512} e^{-3 \beta \eta} h^{3} K 1^{2} \beta^{3}+\frac{7}{256} e^{-\beta \eta} h^{3} K 1^{2} \beta^{3}-\frac{3 h^{3} K 1^{3} \beta^{5}}{1024}$

$-\frac{17 \mathrm{e}^{-7 \beta \eta} \mathrm{h}^{3} \mathrm{~K} 1^{3} \beta^{5}}{12288}+\frac{5 \mathrm{e}^{-5 \beta \eta} \mathrm{h}^{3} \mathrm{~K} 1^{3} \beta^{5}}{4096}+\frac{\mathrm{e}^{-3 \beta \eta} \mathrm{h}^{3} \mathrm{~K} 1^{3} \beta^{5}}{4096}$

$+\frac{35 \mathrm{e}^{-\beta \eta} \mathrm{h}^{3} \mathrm{~K} 1^{3} \beta^{5}}{12288}+\frac{3}{2} \mathrm{e}^{-\beta \eta} \mathrm{h} \eta+\frac{3}{8} \mathrm{e}^{-\beta \eta} \mathrm{h}^{2} \eta+\frac{1}{16} \mathrm{e}^{-\beta \eta} \mathrm{h}^{3} \eta$

$-\frac{3}{64} e^{-3 \beta \eta} h^{2} K 1 \eta+\frac{5}{64} e^{-\beta \eta} h^{2} K 1 \eta+\frac{37 e^{-4 \beta \eta} h^{3} K 1 \eta}{1440}$

$-\frac{15}{256} e^{-3 \beta \eta} h^{2} K 1 \eta-\frac{1}{96} e^{-2 \beta \eta} h^{3} K 1 \eta+\frac{679 e^{-\beta \eta} h^{3} K 1 \eta}{11520}$

$-\frac{5 e^{-3 \beta \eta} h^{3} \eta}{768 \beta^{6}}-\frac{7 e^{-2 \beta \eta} h^{3} \eta}{576 \beta^{6}}-\frac{e^{-\beta \eta} h^{3} \eta}{2304 \beta^{6}}-\frac{e^{-2 \beta \eta} h^{2} \eta}{8 \beta^{4}}-\frac{3 e^{-\beta \eta} h^{2} \eta}{32 \beta^{4}}$

$+\frac{5 e^{-3 \beta \eta} h^{3} \eta}{384 \beta^{4}}+\frac{e^{-2 \beta \eta} h^{3} \eta}{48 \beta^{4}}+\frac{e^{-\beta \eta} h^{3} \eta}{384 \beta^{4}}-\frac{3 e^{-\beta \eta} h \eta}{4 \beta^{2}}+\frac{e^{-2 \beta \eta} h^{2} \eta}{4 \beta^{2}}$

$+\frac{e^{-\beta \eta} h^{2} \eta}{8 \beta^{2}}+\frac{e^{-2 \beta \eta} h^{3} \eta}{48 \beta^{2}}+\frac{e^{-\beta \eta} h^{3} \eta}{96 \beta^{2}}-\frac{37 e^{-4 \beta \eta} h^{3} K 1 \eta}{2880 \beta^{2}}$

$+\frac{5 e^{-3 \beta \eta} h^{3} K 1 \eta}{384 \beta^{2}}+\frac{e^{-2 \beta \eta} h^{3} K 1 \eta}{144 \beta^{2}}-\frac{23 e^{-\beta \eta} h^{3} K 1 \eta}{5760 \beta^{2}}$

$+\frac{3}{32} e^{-2 \beta \eta} h^{2} K 1 \beta^{2} \eta-\frac{3}{32} e^{-\beta \eta} h^{2} K 1 \beta^{2} \eta+\frac{9}{128} e^{-3 \beta \eta} h^{3} K 1 \beta^{2} \eta$

$-\frac{9}{128} e^{-\beta \eta} h^{3} K 1 \beta^{2} \eta-\frac{5 e^{-5 \beta \eta} h^{3} K 1^{2} \beta^{2} \eta}{1024}+\frac{11 e^{-3 \beta \eta} h^{3} K 1^{2} \beta^{2} \eta}{3072}$

$+\frac{5 e^{-\beta \eta} h^{3} K 1^{2} \beta^{2} \eta}{1536}+\frac{5}{512} e^{-5 \beta \eta} h^{3} K 1^{2} \beta^{4} \eta-\frac{3}{512} e^{-3 \beta \eta} h^{3} K 1^{2} \beta^{4} \eta$

$-\frac{1}{256} e^{-\beta \eta h^{3} K 1^{2} \beta^{4} \eta}-\frac{e^{-2 \beta \eta} h^{3} \eta^{2}}{96 \beta^{5}}-\frac{e^{-\beta \eta} h^{3} \eta^{2}}{192 \beta^{5}}-\frac{3 e^{-\beta \eta} h^{2} \eta^{2}}{32 \beta^{3}}$

$+\frac{e^{-2 \beta \eta} h^{3} \eta^{2}}{24 \beta^{3}}+\frac{3 e^{-\beta \eta} h^{2} \eta^{2}}{8 \beta}-\frac{e^{-2 \beta \eta} h^{3} \eta^{2}}{24 \beta}+\frac{e^{-\beta \eta} h^{3} \eta^{2}}{12 \beta}$

$-\frac{3 e^{-3 \beta \eta} h^{3} K 1 \eta^{2}}{512 \beta}+\frac{7 e^{-\beta \eta} h^{3} K 1 \eta^{2}}{1536 \beta}-\frac{3}{8} e^{-\beta \eta} h^{2} \beta \eta^{2}-\frac{1}{8} e^{-\beta \eta} h^{3} \beta \eta^{2}$

$+\frac{3}{128} e^{-3 \beta \eta} h^{3} K 1 \beta \eta^{2}-\frac{5}{384} e^{-\beta \eta} h^{3} K 1 \beta \eta^{2}-\frac{3}{128} e^{-3 \beta \eta} h^{3} K 1 \beta^{3} \eta^{2}$

$+\frac{1}{128} e^{-\beta \eta} h^{3} K 1 \beta^{3} \eta^{2}-\frac{1}{32} e^{-\beta \eta} h^{3} \eta^{3}-\frac{e^{-\beta \eta} h^{3} \eta^{3}}{384 \beta^{4}}+\frac{e^{-\beta \eta} h^{3} \eta^{3}}{64 \beta^{2}}$

$+\frac{1}{48} e^{-\beta \eta} h^{2} \beta^{2} \eta^{2}$ 